\title{
Erratum: Sigma and pion decays of mixed heavy hybrids [Phys. Rev. D 83, 094004 (2011)]
}

\author{
Leonard S. Kisslinger® and Dara J. Krute
}

(A) (Received 13 June 2020; published 16 July 2020)

\begin{abstract}
We correct 5 typos and add important information for our Phys. Rev. D 83, 094004 (2011) article. The solutions to the equations obtained using QCD sum rules, the results, and the conclusions in our original article are not modified.
\end{abstract}

DOI: 10.1103/PhysRevD.102.019901

(1) In the Introduction, line 9, "vs." should be "to" and line 10 "vs" should be "vs."

(2) In the 7th sentence in Sec. II after Fig. 1 " $G-\pi$ coupling vanishes." should be "there is no $G-\pi$ coupling."

(3) In the first sentence after Fig. 1 "external pion field"should be "charm quark and anticharm quark"

(4) After Eq. (6), which should end with a "," rather than a ".", add "with $M_{c} \simeq 1.3 \mathrm{Gev}$ the mass of a charm quark".

(5) The results and conclusions of the original article are not affected. 